\title{
Polución por material particulado fino (PM 2,5) incrementa las hospitalizaciones por insuficiencia cardiaca
}

\author{
Pablo Castro ${ }^{1}$, Jeanette Vera ${ }^{2}$, Luis Cifuentes ${ }^{1}$, Gregory Wellenius ${ }^{3}$, Hugo Verdejo ${ }^{1}$, Luis Sepúlveda ${ }^{2}$, \\ José Luis Vukasovic ${ }^{2}$, Silvana Llevaneras ${ }^{1}$, en representación grupo ICARO. \\ 1) Pontificia Universidad Católica de Chile. \\ 2) Universidad de Chile. \\ 3) Beth Israel Deaconess Medical Center, USA.
}

\section{Resumen:}

Antecedentes: Estudios recientes han reportado una asociación entre la contaminación ambiental por material particulado (PM) y el riesgo de hospitalizaciones de pacientes con insuficiencia cardiaca (IC). La región metropolitana de nuestro país constituye un área geográfica en la cual la contaminación es especialmente relevante, asociándose a incrementos periódicos en la morbimortalidad por causa respiratoria. Sin embargo el efecto de la polución por PM en la morbilidad de pacientes con IC no ha sido evaluado en forma sistemática. Objetivo: Evaluar la asociación entre el PM fino y las hospitalizaciones por IC descompensada en hospitales pertenecientes al registro ICARO del área metropolitana.

Métodos: Estudio prospectivo. Entre enero 2002 a diciembre de 2008 se recolectaron las fichas médicas de 529 pacientes residentes de Santiago hospitalizados por IC descompensada. Las variables meteorológicas y de contaminación fueron obtenidas de la red MACAM. Para estudiar la asociación entre las hospitalizaciones y los niveles de contaminación (PM10 y PM2,5), se aplicó un diseño de Casos cruzados estratificado por tiempo (Time-stratified Case-crossover), controlando por temperatura y punto de rocío. El impacto de los niveles de contaminación en el número de hospitalizaciones se evaluó asumiendo una latencia en el efecto de la polución de 0 a 10 días.

Resultados: La edad media de la población en estudio fue de 73.8 años. La etiología más frecuente fue isquémica (27\%) e hipertensiva (27\%). Un $73.2 \%$ de los pacientes eran hipertensos y $32.6 \%$ tenían antecedentes de diabetes mellitus. Se observó un aumento en la admisión por IC descompensada en pacientes de ambos sexos, menores de 74 años, con desde un $22.7 \%$ ( $\mathrm{p}=0.03$ ) por cada incremento en $10 \mathrm{ug} / \mathrm{m} 3$ de PM2.5 calculado con media móvil, cuatro días después de la exposición hasta un $44.8 \%(\mathrm{p}=0.006)$ a 10 días de la exposición. Los pacientes con antecedentes de diabetes mellitus fueron más susceptibles, observándose en ellos un aumento de un $18.8 \%(\mathrm{p}=0.035)$ de las hospitalizaciones por IC por cada incremento de $10 \mathrm{ug} / \mathrm{m} 3$ de PM2.5, ocho días después de la exposición. Los pacientes hipertensos de ambos sexos, menores de 74 años, también mostraron ser suceptibles, con un 22.8\% (2.1-43.5)\% con día 5 de la exposición. Mostrando que este efecto puede ser modificado según sexo y edad.

Conclusión: Nuestros resultados sugieren que pacientes con IC con antecedente de diabetes mellitus e hipertensos, son especialmente susceptibles a la exposición a material particulado, fino y presentan un alto riesgo de hospitalización en relación con incrementos del PM2,5, siendo un factor modificador el sexo y la edad del paciente Los mecanismos involucrados en este fenómeno no han sido estudiados. 


\section{Small particle air pollution (PM2.5) leads to an increase in hospitalization rate for congestive heart failure}

Background: Recent studies have reported an increase risk of hospitalization in patients with congestive heart failure (CHF) in association with air pollution by small particles. The Metropolitan region in Chile is characterized by high pollution indexes which are related to increased mortality from respiratory diseases. No systematic evaluation of the effect of particle pollution upon morbidity in patients with $\mathrm{CHF}$ is available.

Aim. To evaluate the association between fine particle pollution and hospitalization rate for decompensated $\mathrm{CHF}$ in hospitals participating in the ICARO registry of CHF in the Metropolitan area of Santiago.

Methods. In a prospective design the clinical records of 529 patients who were hospitalized for decompensated CHF from Jan 2002 to Dec 2008 were analyzed. Meteorological and pollution indexes were obtained from de MACAM monitoring network. A time stratified case cross-over design was used to study the association between hospitalization rate and pollution indexes (PM10 and PM25. Data was controlled for temperature and "punto de rocío". A 0 to 10 day latency period was es-

\section{Introducción:}

La insuficiencia cardíaca (IC) es un problema creciente de salud pública debido al envejecimiento de la población, mayor prevalencia de factores de riesgo como hipertensión arterial y diabetes y desarrollo de enfermedad ateroesclerótica isquémica. En forma concomitante, la sobrevida al infarto al miocardio ha mejorado con la utilización de terapias de reperfusión y han existido avances en el tratamiento de la IC que disminuyen la progresión de la enfermedad y la muerte atribuible a arritmias ${ }^{1}$. Esta epidemia de IC se traduce en elevados costos en salud, siendo una gran proporción de estos atribuibles al gasto que ocurre durante la hospitalización. La población de pacientes con IC y edad avanzada es especialmente vulnerable a descompensación y rehospitalizaciones ${ }^{2-4}$.

Existe evidencia que la contaminación ambiental aumenta en forma significativa la morbimortalidad de la población general. En los últimos años se ha reconocido efectos tóxicos sobre la salud cardiovascular ${ }^{5-11}$. Es así como la exposición a elevadas concentraciones de material particulado (MP) aumenta el riesgo de eventos isquémicos agudos, altera la función autonómica e timated to evaluate the influence of pollution on hospitalization rate.

Results. The mean age of patients was 73.8 years. Etiologies for $\mathrm{CHF}$ included ischemic heart disease (27\%) and hypertensive heart disease (27\%) $.73 .2 \%$ of patients were hypertensives and $32.6 \%$ had evidence of DM. Hospitalization rate for $\mathrm{CHF}$ in men or women $>74$ years of age increased from $22.7 \% 4$ days after exposure to $44.8 \% 10$ days after exposure $(\mathrm{p}=0.006)$. Diabetic patients were more susceptible to hospitalization with an $18 \%$ increased rate for each 10ug/m3 PM2.5 concentration at 8 days after exposure. Male and female hypertensive patients $<74$ years of age were also susceptible with a $28 \%(2.1$ to $43 / 5 \%, C I)$ increase in hospitalization rate at 5 days after exposure.

Conclusion: Patients with CHF who are diabetics or hypertensives are at increased risk of hospitalization for HF decompensation in relation to exposure to air pollution by PM2.5. Sex and age influence the risk. Mechanisms underlying these effects are not yet elucidated.

incrementa el riesgo de arritmias ${ }^{12}$. Estudios más recientes han reportado una asociación entre contaminación ambiental por MP y riesgo de hospitalizaciones de pacientes con IC.

El MP es una mezcla heterogénea de sólidos y partículas líquidas suspendidas en el aire, que continuamente están variando en tamaño y composición química en el espacio y tiempo. Las emisiones naturales, como antropogénicas, contribuyen al aumento, siendo estas partículas con composiciones de heterogéneas, que van desde materiales orgánicos e inorgánicos ${ }^{13}$. Hay definiciones establecidas para identificar y evaluar la cantidad de este MP en el aire.

La legislación chilena, ha definido y normado, sobre el material particulado grueso o PM10, como las partículas que miden menos de $10 \mathrm{um}$. Pero, en los últimos años, estudios epidemiólogos y la EPA han identificado y han decidido evaluar los efectos en la salud del material particulado fino o PM2,5, definiéndola como las partículas cuyo diámetro aerodinámico medio es de $<$ 2,5um, ya que este tiene el potencial de entrar directamente, a los alveolos y penetrar por los tejidos del epitelio interno.

El año 1996, la Región Metropolitana, fue declara- 
da Zona Saturada por 4 contaminantes: ozono (O3), material particulado respirable (PM10), partículas en suspensión (PTS) y monóxido de carbono (CO) (Decreto Supremo $\mathrm{N}^{\circ}$ 131). Las condiciones, especiales de la cuenca geográfica, patrones de viento, las condiciones meteorológicas cambiantes durante el año, son factores que suman para que los patrones de contaminación, comparado a otras regiones del mundo, sean especialmente relevante ${ }^{14}$. Estudios previos, han asociado significativamente el incremento de la mortalidad ${ }^{15,16}$ y morbilidad cardiovascular ${ }^{17,18}$ en Santiago, por exposición a niveles de material particulado grueso y fino.

Sin embargo, el efecto de la polución por MP en la morbilidad de pacientes con IC no ha sido evaluado en forma sistemática. Más aún en la población de pacientes con IC existen distintas comorbilidades que podrían influir en la vulnerabilidad al efecto de la contaminación. Es así, como se ha observado que Individuos con diabetes mellitus están expuestos a un mayor riesgo de ser hospitalizados por enfermedades cardíacas durante períodos de mayor contaminación ambiental ${ }^{19}$. En ellos, la exposición prolongada a MP2,5 se ha asociado a mayor mortalidad en relación a la población general postulándose el gatillamiento de fenómenos inflamatorios y de estrés oxidativo entre otros ${ }^{20-21}$.

En el año 2002 y a instancias de la Sociedad Chilena de Cardiología se creó el Registro Nacional de IC (registro ICARO) que reúne las características clínicas, formas de presentación, comorbilidades, evolución intrahospitalaria y exámenes de laboratorio de pacientes hospitalizados por IC. El objetivo del presente trabajo es evaluar la asociación entre el MP fino y las hospitalizaciones por IC descompensada en hospitales del registro pertenecientes al área metropolitana. Esto nos parece relevante debido a que la identificación de la población más susceptible puede contribuir a la implementación de medidas preventivas y potenciar el desarrollo de políticas públicas efectivas en el manejo de la contaminación ambiental.

\section{Métodos:}

\section{Obtención de datos:}

Para el presente estudio se consideraron 530 pacientes con residencia en Santiago admitidos entre enero 2002 y mayo 2009 con el diagnóstico de IC descompensada en 10 centros médicos de Santiago, Chile. Como criterio diagnóstico de IC se consideró: 1. Presencia de síntomas característicos y 2. Evidencia objetiva de disfunción cardíaca mediante ecocardiograma o radiografía de tórax. En casos dudosos se incluyó como criterio la respuesta al favorable tratamiento de IC. Se excluyeron del registro pacientes que debutaron con shock cardiogénico debido a síndrome coronario agudo. Las característica clínicas, ecocardiográficas y de laboratorio fueron registradas al momento de la admisión y al alta.

Los datos meteorológicos y de contaminación se obtuvieron de la red de monitoreo MACAM (Red de Monitoreo Automática de la Calidad del Aire Metropolitano). Los valores diarios de temperatura y humedad relativa se emplearon para el cálculo del punto de rocío, que corresponde a la temperatura a la que empieza a condensarse el vapor de agua en el aire. De los datos de polución se consideró el material particulado fino (PM2,5) y grueso (PM10) . Para todos los casos se consideró el promedio diario en 24 horas como valor representativo.

\section{Diseño:}

Para estudiar la asociación entre las hospitalizaciones y los niveles de contaminación (PM10 y PM2,5), se aplicó en un diseño de Casos Cruzados Estratificado por Tiempo (Timestratified Case-crossover, TSCC). Este diseño, deriva del caso-control y valora el riesgo de eventos a corto o mediano plazo asociados a una exposición intermitente o inusual ${ }^{22,23}$. Metodológicamente el diseño plantea varias ventajas. 1) Los pacientes seleccionados sirven de control para ellos mismos, lo que permite controlar el efecto de posibles variables contundentes, propias de cada paciente (edad, sexo, etnia, hábito tabáquico, medicamentos), o derivadas del período de tiempo estudiado. 2) Puede aplicarse en un relativo número pequeño de pacientes, a diferencia del enfoque tradicional (Series de tiempo). 3) Elimina el sesgo de selección del paciente. Esto, debido a que en el diseño TSCC el paciente constituye un "caso" al momento de su ingreso, y constituye un "control" en todas las oportunidades en las cuales se verificó exposición a riesgo (en este caso, contaminación por PM2,5 y PM10) y en las cuales no se verificó una hospitalización. Dada las características del diseño estratificado por tiempo, y para controlar el efecto estacional, se consideró como control un período de tiempo del mismo mes.

Se realizaron 3 análisis por separado:1) Población total de pacientes que ingresaron por IC 2) Estratificación por comorbilidad conocida (diabetes, hipertensión y asociación de ambas), 3) Estratificación por etiología (isquémica o hipertensiva). Una vez identificados los posibles grupos de susceptibles, se evaluó el efecto modificador de sexo y edad.

\section{Modelo Base:}


Polucion por material particulado fino $(\mathrm{pm} 2,5)$ incrementa las hospitalizaciones por insuficiencia cardiaca Castro $P$ et al.

\begin{tabular}{|c|c|c|c|c|c|c|c|}
\hline \multirow{3}{*}{ Par metro } & \multicolumn{6}{|c|}{$\begin{array}{l}\text { Tabla 1: Valores meteorol gicos y de los contaminantes, } \\
\text { Per odo } 2003 \text { a } 2008 .\end{array}$} & \\
\hline & N & Media & Desviaci $n$ & & & \multicolumn{2}{|c|}{ Percentiles } \\
\hline & ilas & & Est ndar & M nimo & M ximo & 5 & 95 \\
\hline PM10 (ug/m3) & 2168 & 69,5 & 30,2 & 6,8 & $211,4^{\star \star}$ & 31,4 & 130,8 \\
\hline PM2, (ug/m3) & 2190 & 32,1 & 15,9 & 4,9 & $108,4^{* \star *}$ & 14,0 & 64,3 \\
\hline Temperatura (C) & 2192 & 16,2 & 4,9 & 3,2 & 26.9 & 8,3 & 23,4 \\
\hline Punto de Roci (C) & 2149 & 8,2 & 3,4 & $-3,9$ & 16,4 & 2,1 & 13,3 \\
\hline
\end{tabular}

* Promedios diarios de 24 horas.

** Valor 11/05/2007

*** Valor 31/05/2003

El modelo base para estudiar a asociación fue representado en una ecuación condicional logística, cuyas variables son: 1) Caso, 2) Valor del promedio diario del material particulado, controlado por el promedio diario la temperatura y punto de rocío ajustadas por natural spliens de 2 dof para en cada caso. La hipótesis evaluada fue el efecto de los niveles del material particulado a corto plazo, desde 0 días post exposición (lag 0) hasta un máximo de 10 días (lag10). En forma paralela, se estimó la influencia del promedio móvil de contaminación desde el día 1 (mm1) hasta 10 días post exposición (mm10).

Nosotros sometimos a prueba, la influencia de los niveles de material particulado en el corto plazo, es decir, cuanto al día de ingreso al hospital (Lag 0), hasta los 10 días previos de los niveles de contaminación PM10 y PM 2,5 (lag 10). Paralelamente, se estimó la influencia del promedio móvil de la contaminación, desde el día de ingreso al día anterior (promedio móvil 1: ma1) hasta los 10 días de exposición (promedio móvil 10: ma10). Los hallazgos significativos se representaron en gráficos ad-hoc. Todos los análisis se realizaron en software $\mathrm{R}$ versión 2.9, paquete Epicalc.

Aspectos Éticos.

No existió intervención farmacológica sobre la evolución de los pacientes. El grupo Investigador se encargó de velar la confidencialidad de la identidad de los pacientes, según normas de Buenas Prácticas Clínicas, Comité de Ética Sociedad Chilena de Cardiología y los Comités de Ética locales de los centros participantes.

\section{Resultados:}

\section{Características clínicas}

Durante el período de estudio se incorporaron 529 pacientes. La edad media de la población fue de 73,8 años, $53.3 \%$ (283) de sexo masculino. Un $49,4 \%$ de la población (260 pacientes) correspondía a menores de 74 años.
El antecedente de hipertensión arterial estaba presente en la mayoría de los pacientes participantes (388 individuos, $73,1 \%$ ), siendo más común en la población mayor de 74 años (56,2 vs. $43,8 \%)$.

$\mathrm{El}$ antecedente de diabetes mellitus (DM) estaba presente en 170 pacientes $(32 \%)$, sin diferencias significativas entre grupos etarios $(52,7$ vs $47,3 \%$ para mayores y menores de 74 años, respectivamente). Un $27,7 \%$ (170) de los pacientes presentaba ambas patologías.

En el grupo de estudio, la etiología de la IC fue predominantemente isquémica $(29,1 \%)$ e hipertensiva $(29 \%)$. Un 7,8\% de los pacientes tenía una causa valvular para su enfermedad. Un 16,3\% de los pacientes ingreso a causa de infecciones respiratorias (neumonía).

Valores meteorológicos y niveles de contaminación (tabla 1): El valor promedio de la temperatura fue de un 16,2 ${ }^{\circ} \mathrm{C}$ (DS: 4,99), el Punto de Rocío fue de un $8,2^{\circ} \mathrm{C}$ (DS: $3,4)$. El promedio para el material particulado PM2,5, fue de un 32,6 ug/m3, (DS: 15,9) PM10, fue de un 69,5 ug/ m3, (DS: 30,2). Los valores meteorológicos de relevancia se detallan en la Tabla 1. Para los siguientes modelos, los valores representan el incremento en el riesgo de eventos asociados a un aumento en $10 \mathrm{ug} / \mathrm{m} 3$ de la concentración del polutante.

Efecto de la exposición a material particulado en el riesgo de hospitalización por IC descompensada: La exposición a PM2,5 no se asoció a un incremento significativo en el riesgo de hospitalización en la población general estudiada. Sin embargo, la estratificación por edad mostró que los pacientes menores a 74 años mostraban un incremento significativo de riesgo de hospitalización luego de la exposición (desde el día 4 con un 14,8\% (0,4-31,2\%), en PM2,5, $\mathrm{p}=0,04$, aumentando el día 9, a un 19,6\% (4,8$36,6 \%)$, PM25, $\mathrm{p}=0,008)$, calculado este riesgo con la media móvil, se observan riesgos mayores (desde el día 5 con un riesgo de 22,7 (1,6-48,2)\%, PM2,5, p=0,003 aumentando para el día 10, a 44,8\%(11-89) $\mathrm{p}=0,0006)$. De igual forma, la estratificación por sexo mostró que los pacientes de sexo masculino eran consistentemente 


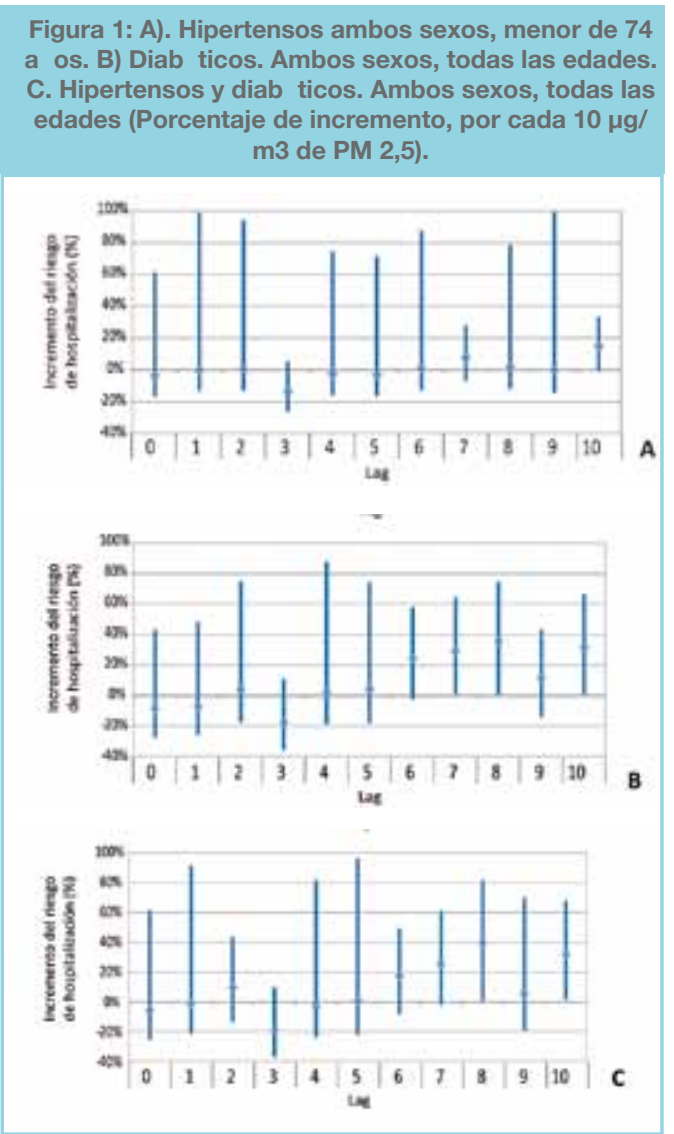

más susceptibles a la exposición única (lag) o acumulativa (promedio móvil) de PM2,5, asociándose a un incremento máximo del riesgo a la acumulación de niveles altos de contaminación 22,8\% (5,1-41,0\%), $\mathrm{p}=0,01$, lag 5).

\section{Efecto de la exposición a material particulado según comorbilidad:}

Hipertensión: El antecedente de hipertensión incrementó el riesgo de hospitalización luego de exposición a material particulado en individuos menores de 74 años, tanto para el material particulado fino $(8,8 \%$ (CI: $2,6-$ $35 \%$ ), con PM2,5 lag 4, p=0,02 y 20,5\% (CI: 4,9-37\%), con PM2,5 lag 5,p=0,01) y grueso 8,6\% (CI: 0,4-17\%) para PM10 lag 5, p=0,04). Los resultados significativos se muestran en la Figura 1 y 2. De manera similar, el efecto acumulativo evaluado mediante el promedio móvil diario de 0 a 5 días confirmó un incremento de riesgo de hospitalización en este subgrupo frente a la exposición a material particulado fino (Incremento $33.4 \%$ (CI: 0.4-69\%) para PM2,5, p=0,05) y grueso (Incremento 18,4\% (CI:1,1-36\%) para PM10, p=0,04). En pacientes mayores de 74 años sólo se observó un in-

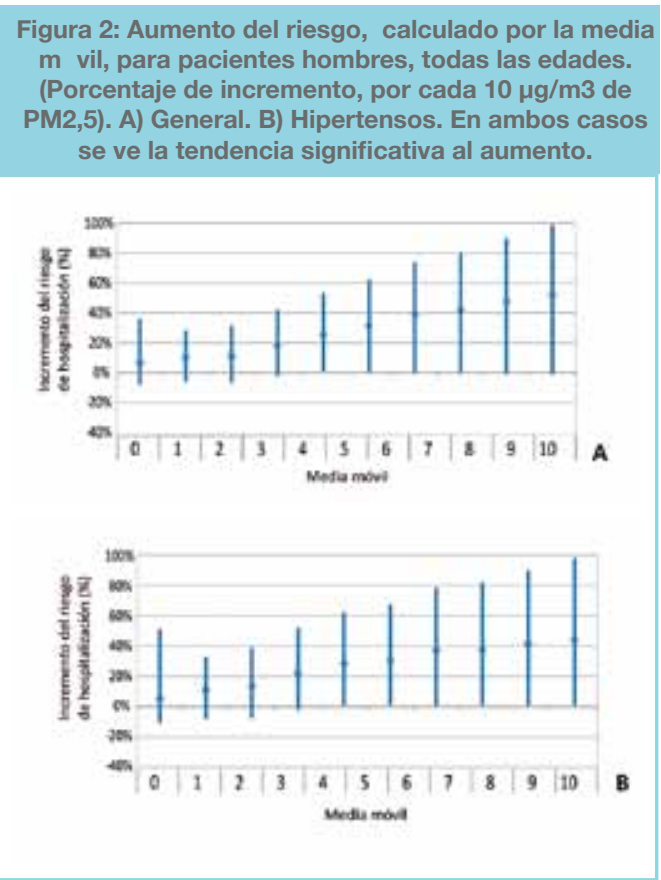

cremento en el riesgo de 13,8\% (CI: 0,0-28\%) al día 10 post exposición a PM25 (p=0,05). (OJO: 25 o 2,5 ¿??) Al evaluar los grupos estratificados por sexo, el riesgo de hospitalización post exposición a material particulado fino afecta principalmente al sexo masculino, con un incremento de $17,1 \%(2,7-32 \%), p=0,02, y 16,5 \%$ (1,9$32 \%), \mathrm{PM} 2,5, \mathrm{p}=0.03$ para el día 4 y 5 post exposición, respectivamente. La exposición a material particulado grueso se asoció a un perfil de riesgo similar, aunque menor cuantía (Incremento 10\% (CI 2,3 - 28\%) para el día 4 post exposición) (Figura 2 y 3 ).

Diabetes mellitus: Los pacientes diabéticos constituyen un grupo particularmente vulnerable en nuestra serie. Los pacientes incluidos presentaron un incremento en el riesgo de hospitalización post exposición tanto a material particulado fino (incremento 17,2\% (CI: 1,2$34 \%$ ) lag 8), (tabla 2 y Figura 1), como grueso (incremento 9,44\% (CI 0,7-18\%), lag 8. La estratificación por edad evidenció que el incremento de riesgo fue más evidente en los pacientes diabéticos de más de 74 años (incremento 25,5\% (CI: 1,9-50\%) para PM2,5 lag 7, $\mathrm{p}=003$ y $30,5 \%$ (CI 6,0-57\%), lag $8, \mathrm{p}=0,02)$. Al igual que en los análisis anteriores, la exposición a material particulado grueso se asoció a un incremento de riesgo 


\begin{tabular}{|c|c|c|c|c|c|c|}
\hline \multirow[t]{2}{*}{ D a } & \multirow[t]{2}{*}{ Media (ug/m3) } & \multirow{2}{*}{$\begin{array}{l}\text { Desviaci } \mathrm{n} \\
\text { Est ndar }\end{array}$} & \multirow[t]{2}{*}{ Max } & \multirow[t]{2}{*}{ Min } & \multirow[t]{2}{*}{ Rango } & Percentil \\
\hline & & & & & & $5,0 \quad 95,0$ \\
\hline 0 & 36,4 & 17,6 & 83,3 & 9,8 & $73,515,4$ & 68,6 \\
\hline 1 & 36,1 & 17,1 & 79,1 & 8,6 & $70,614,0$ & 67,1 \\
\hline 3 & 34,0 & 16,0 & 75,1 & 10,7 & $64,313,9$ & 66,2 \\
\hline 8 & 3,7 & 18,5 & 94,2 & 11,0 & $83,214,1$ & 72,7 \\
\hline
\end{tabular}

menor al observado para PM2,5 (incremento 13\% (CI: 1,7-26\%) para PM10, lag 7 y 14,6\% (CI 1.6-28\%) para PM10, lag $8, \mathrm{p}=0,03$ por $10 \mathrm{ug} / \mathrm{m} 3$ polutante).

La estratificación por sexo mostró hallazgos similares a los anteriormente descritos, mostrando un incremento de riesgo más evidente en población masculina (incremento de riesgo $25,2 \%$ (CI: 0,3-48\%), lag $6 \mathrm{p}=0,03 \mathrm{y}$ $28,8 \%$ (CI: $0.5-54 \%), \operatorname{lag} 8 \mathrm{p}=0.03$ para PM25 y $17,7 \%$ (CI: $4.8-31 \%)$, lag $8, \mathrm{p}=0.01$ para PM10).

La asociación de ambas comorbilidades (DM e hipertensión) se asoció a una susceptibilidad más evidente a la exposición a material particulado fino (incremento del riesgo $17,9 \%$ (CI:1,0-36)\% para PM2,5, lag 8, $\mathrm{p}=0,04)$ y grueso (incremento $11,1 \%$ (CI:2-21\%) para PM10, lag 8, p=0,002).

\section{Discusión:}

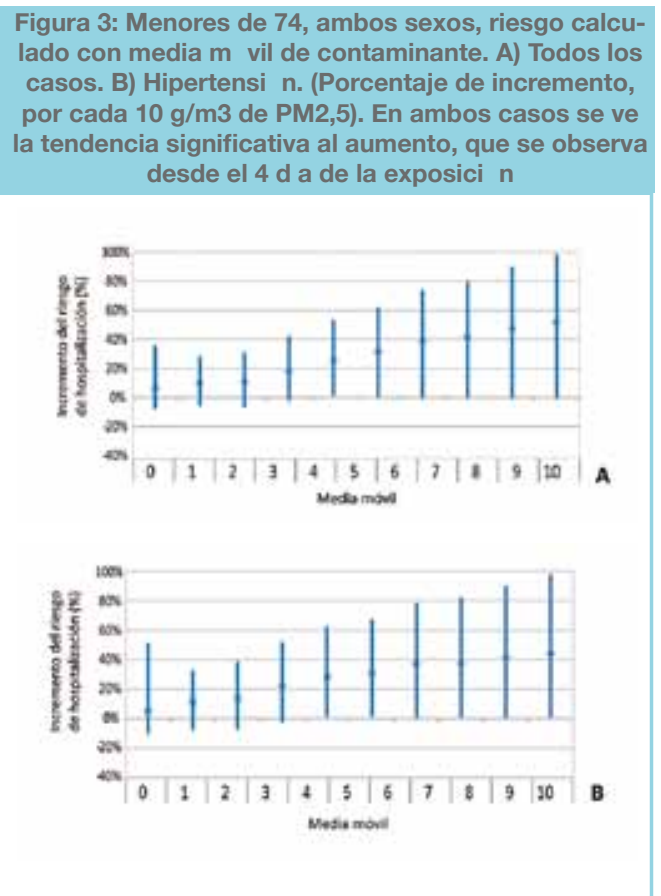

El principal hallazgo de este estudio es que la contaminación ambiental determinada por material particulado fino (MP2,5) y grueso (PM10) tiene un efecto deletéreo en pacientes con IC, asociándose a un incremento en el riesgo de hospitalización por descompensación de la IC. Este efecto es significativo en un subgrupo de pacientes vulnerables, en particular hombres de menos de 74 años con antecedentes de hipertensión arterial, pacientes diabéticos añosos y pacientes con ambas comorbilidades (diabéticos e hipertensos). En general, el efecto de la contaminación en este grupo vulnerable se hace manifiesto luego de alrededor desde una 1 semana post máxima exposición. No se logró identificar una asociación significativa con la etiología de la IC o el mecanismo de la descompensación.

En nuestro país, la contaminación ambiental en el área metropolitana y algunas otras ciudades como Temuco constituye un problema relevante que tradicionalmente se ha relacionado con descompensaciones del tipo respiratorias de predominio estacional. Recientemente, el impacto de la polución ambiental en otros sistemas ha sido evaluado; diversos estudios han demostrado un posible efecto de la contaminación en eventos cardiovasculares. En general, en pacientes con IC se ha observado mayor riesgo de hospitalizaciones, aún cuando los resultados dependen del área geográfica estudiada, nivel de contaminación y población de pacientes estudiada ${ }^{23}$. Este es el primer estudio a nivel nacional que demuestra un impacto negativo de la contaminación ambiental por material particulado fino (MP2,5) y grueso (PM10) en individuos portadores de IC. La exposición a ambos tipos de polutantes resultó relevante, aún cuando es más evidente en relación a MP2,5. Este grupo de contaminantes corresponde a partículas finas con un diámetro aerodinámico de menos de $2,5 \mu \mathrm{m}$; la composición de las partículas es variable dependiendo del área geográfica, condiciones meteorológicas y fuentes contaminantes (refinerías, instalaciones procesadoras de metales, etc) lo que limita la extrapolación de los resultados de cohortes internacionales. Por otra parte, 
las partículas PM10 provienen de polvo de los caminos, desgaste de neumáticos, combustión de maderas, materiales desprendidos en demoliciones y construcción, operaciones mineras e incendios forestales. En Santiago, y durante el período de estudio, los valores de PM2,5 fueron en promedio 32,1 ug/m3 PM25, un valor tres veces mayor al reportado en ciudades de Estados Unidos, donde fluctúa entre 10 y $11 \mathrm{ug} / \mathrm{m} 3$ de PM2, $5^{23}$, lo que puede contribuir a explicar el impacto del material particulado fino en la descompensación de estos individuos en nuestra cohorte. Estos resultados son similares a los previamente reportados por otros investigadores en Canadá, Australia y Nueva Zelanda (Burnet, 1999, Barnett, 2006), aún cuando la latencia del efecto en nuestra cohorte es significativamente mayor, lo que pone en evidencia nuestra pobre comprensión de los mecanismos fisiopatológicos involucrados.

Los mecanismos subyacentes al efecto de la contaminación en el sistema cardiovascular descritos en la literatura son numerosos. En modelos experimentales se ha observado inflamación y fibrosis miocárdica ${ }^{24}$ hallazgos han sido confirmados en estudios en humanos que han demostrado que la exposición a MP se asocia a una respuesta inflamatoria evidenciada por incrementos de en los niveles de proteína $\mathrm{C}$ reactiva $\mathrm{y}$ otros mediadores inflamatorios, lo que determina hipercoagulabilidad, disfunción endotelial, vasoconstricción e isquemia miocárdica ${ }^{25-30}$.

La inhalación de MP se ha asociado a inducción de estrés oxidativo en miocardiocitos de rata neonatales y en células endoteliales de rata, hallazgo que se ha confirmado por observaciones en voluntarios sanos y en pacientes con diabetes mellitus tipo $2^{31}$.

Otro posible mecanismo que puede contribuir a la descompensación de pacientes con IC puede depender del sistema nervioso autónomo. La exposición a material particulado se asocia a cambios en la variabilidad de la frecuencia cardíaca (FC), aumento de la FC de reposo e incrementos en la presión arterial, lo que parece depen- der de un aumento en el tono simpático ${ }^{32-34}$. Probablemente, varios de estos mecanismos participan en forma simultánea en pacientes de los grupos más suceptibles. La aparente paradoja del incremento de riesgo en individuos con antecedentes de hipertensión y edad menor a 74 años puede depender de factores no evaluados en este estudio, como diferencias geográficas y socioculturales que influyen en el patrón de exposición a polutantes ${ }^{35,36}$.

Limitación: Con respecto a la magnitud de los intervalos de riesgo observados en Santiago, si bien los resultados son robustos, pensamos que tiene que ver con la cantidad de pacientes que estamos integrando. Si bien el diseño Caso Cruzados (Case-crossover), es un diseño especializado y más robusto del Caso y Control, pero es un diseño, menos preciso para determinar los intervalos de riesgos, comparado con otros diseños epidemiológicos, como es el que se aplica para Series de Tiempo o Time Series, pero necesita una mayor cantidad de pacientes para ser válido aplicar.

En resumen, este estudio demuestra que la contaminación ambiental determinada por material particulado fino (MP2,5) y grueso (PM10) tiene un efecto deletéreo en pacientes con IC, asociándose a un incremento en el riesgo de hospitalización por descompensación de la IC. Este efecto es significativo en un subgrupo de pacientes vulnerables, en particular hombres de menos de 74 años con antecedentes de hipertensión arterial, pacientes diabéticos añosos y pacientes con ambas comorbilidades (diabéticos e hipertensos). En general, el efecto de la contaminación en este grupo vulnerable se hace manifiesto luego de alrededor de una 1 semana post máxima exposición. Estos hallazgos nos parecen relevantes en la identificación de subpoblaciones de mayor riesgo frente a la contaminación. Medidas preventivas y políticas de salud pública debieran considerar estos resultados en el control y prevención de material contaminante en el área metropolitana de Santiago. 


\section{Referencias:}

1. Najafi F, Jamrozik K, Dobson AJ. Understanding the 'epidemic of heart failure': a systematic review of trends in determinants of heart failure. Eur J Heart Fail. 2009; 11:472-9.

2. De Lissovoy G, Fraeman K, Teerlink JR, Mullahy J, Salon J, Sterz $\mathrm{R}$, et al. Hospital costs for treatment of acute heart failure: economic analysis of the REVIVE II study. Eur J Health Econ. 2010; 11: 185-93.

3. Ehrmann Feldman D, Ducharme A, Frenette M, Giannetti N, Michel C, Grondin F, et al. Related to time to admission to specialized multidisciplinary clinics in patients with congestive heart failure. Can J Cardiol. 2009; 25: e347-52

4. Tibaldi V, Isaia G, Scarafiotti C, Gariglio F, Zanocchi M, Bo M, et al. Hospital at home for elderly patients with acute decompensation of chronic heart failure: a prospective randomized controlled trial. Arch Intern Med. 2009; 169: 1569-75

5. Bhatangar A, Environmental cardiology: Studying mechanistic links between pollution and heart disease. Circ Res. 2006; 99: 692705 .

6. Wellenius GA, Schwartz J, Mittleman MA. Particulate air pollution and hospital admissions for congestive heart failure in seven U.S. cities. Am J Cardiol 2006; 97: 404-408.

7. Pope CA 3rd, Burnett RT, Thurston GD, Thun MJ, Calle EE, Krewski D, et al., Cardiovascular mortality and long-term exposure to particulate air pollution: Epidemiological evidence of general pathophysiological pathways of disease. Circulation 2004; 109:.71-77.

8. Brook RD, Franklin B, Cascio W, Hong Y, Howard G, Lipsett M, et al., Air pollution and cardiovascular disease: A statement for health care professionals from the expert panel on population and prevention science of the American Heart Association. Circulation 2004; 109: 2655-2671.

9. Hoffmann B, Moebus S, Möhlenkamp S, Stang A, Lehmann N, Dragano N, et al. and Heinz Nixdorf Recall Study Investigative Group, Residential exposure to traffic is associated with coronary atherosclerosis. Circulation 2007; 116: 489-496.

10. Franchini M, Mannucci PM. Short-term effects of air pollution on cardiovascular diseases: outcomes and mechanisms, J Thromb Haemost 2007: 2169-2174.

11. Román O, Prieto MJ, Mancilla P. Contaminación atmosférica y daño cardiovascular. Rev Med Chil. 2004; 132: 761-7.

12. Chiu HF, Yang CY. Air pollution and emergency room visits for arrhythmias: are there potentially sensitive groups? J Toxicol Environ Health A. 2009; 72: 817-238.

13. Simkhovich BZ, Kleinman MT, Kloner RA. Air pollution and cardiovascular injury epidemiology, toxicology, and mechanisms.
J Am Coll Cardiol. 2008; 52: 719-26.

14. Brook RD, Franklin B, Cascio W, Hong Y, Howard G, Lipsett M, et al. Expert Panel on Population and Prevention Science of the American Heart Association. Air pollution and cardiovascular disease: a statement for healthcare professionals from the Expert Panel on Population and Prevention Science of the American Heart Association. Circulation. 2004; 109: 2655-71.

15. Cifuentes LA, Vega J, Köpfer K, Lave LB. Effect of the fine fraction of particulate matter versus the coarse mass and other pollutants on daily mortality in Santiago, Chile. J Air Waste Manag Assoc. 2000; 50: 1287-98.

16. Ostro BD, Eskeland GS, Sanchez JM, Feyzioglu T. Air pollution and health effects: A study of medical visits among children in Santiago, Chile. Environ Health Perspect. 1999; 107: 69-73.

17. Ostro B, Sanchez JM, Aranda C, Eskeland GS. Air pollution and mortality: results from a study of Santiago, Chile. J Expo Anal Environ Epidemiol. 1996; 6: 97-114.

18. Vera J. Cifuentes LA. Strappa V. Association of Particulate Matter and Hospital Admissions in Santiago, Chile. Epidemiology. 2007; 18: S210-S211

19. Vera J; Cifuentes L. Association of Hospital Admissions for Cardiovascular Causes and Air Pollution (PM10, PM25 and O3) in Santiago, Chile. Epidemiology. 2008; 19: S368.

20. Gold DR. Vulnerability to cardiovascular effects of air pollution in people with diabetes. Curr Diab Rep. 2008; 8: 333-5.

21. Madrigano J, Baccarelli A, Wright R, Suh H, Sparrow D, Vokonas $\mathrm{P}$, et al. Air pollution, obesity, genes and cellular adhesion molecules. Occup Environ Med. 2010; 67: 312-7.

22. Liu L, Ruddy TD, Dalipaj M, Szyszkowicz M, You H, Poon R, et al. Influence of personal exposure to particulate air pollution on cardiovascular physiology and biomarkers of inflammation and oxidative stress in subjects with diabetes. J Occup Environ Med. 2007; 49: 258-65.

23. Janes H, Sheppard L, Lumley T. Case-crossover analyses of air pollution exposure data: referent selection strategies and their implications for bias. Epidemiology. 2005; 16: 717-26.

24. Eduardo Carracedo Martínez, Aurelio Tobías, Marc Sáez, Margarita Taracido Trunk, Adolfo Figueiras. Fundamentos y aplicaciones del diseño de casos cruzados. Gaceta sanitaria: Organo oficial de la Sociedad Española de Salud Pública y Administración Sanitaria, 2009; 23: 161-165.

25. Strappa V, Cifuentes A, Jorquera H. Importance of the population exposure model in the impact of PM and daily mortality. Epidemiology. 2009; 20: S217.

26. Wellenius GA, Schwartz J, Mittleman MA. Particulate Air Pollution and Hospital Admissions for Congestive Heart Failure in Seven United States Cities. Am J Cardiol. 2006; 97: 404-8. 
27. Kodavanti UP, Moyer CF, Ledbetter AD, Schladweiler MC, Costa DL, Hauser R, et al. Inhaled environmental combustion particles cause myocardial injury in the Wystar Kyotorat. Toxicol rat. Toxicol Sci. 2003; 71: 237-45.

28. Pope CA 3rd, Hansen ML, Long RW, Nielsen KR, Eatough NL, Wilson WE, et al. Ambient particulate air pollution, heart rate variability, and blood markers of inflammation in a panel of elderly subjects. Environ Health Perspect 2004; 112: 339-45.

29. Mills NL, Törnqvist H, Gonzalez MC, Vink E, Robinson SD, Söderberg $\mathrm{S}$, et al. Ischemic and thrombotic effects of dilute dieselexhaust inhalation in men with coronary heart disease. $\mathrm{N}$ Eng $\mathbf{J}$ Med 2007; 357: 1075-82.

30. Peters A, Fröhlich M, Döring A, Immervoll T, Wichmann HE, Hutchinson WL, et al. Particulate air pollution is associated with an acute phase response in men. Eur Heart J 2001; 22: 1198-204.

31. Rückerl R, Greven S, Ljungman P, Aalto P, Antoniades C, Bellander T, et al.Air pollution and inflammation (interleukin-6, C-reactive protein, fibrinogen) in myocardial infarction survivors. Environ Health Perspect 2007; 115: 1072- 80.
32. O'Neill MS, Veves A, Zanobetti A, Sarnat JA, Gold DR, Economides PA, et al. Diabetes enhances vulnerability to particulate air pollution-associated impairment in vascular reactivity and endothelial function. Circulation 2005; 111: 2913-20.

33. Brook RD, Brook JR, Urch B, Vincent R, Rajagopalan S, Silverman F. Inhalation of fine particulate air pollution and ozone causes acute arterial vasoconstriction in healthy adults. Circulation 2002; 105: 1534-6.

34. Jalil J, Dumas E, Cifuentes L, Ocaranza MP, Chamorro G, Padilla I. Desbalance autonómico y procoagulación debido a contaminación atmosférica en Santiago. Rev Chil Cardiol 2003; 22: 223232.

35. Kleiger RE, Miller JP, Bigger JT Jr, Moss AJ. Decreased heart rate variability and its association with increased mortality after acute myocardial infarction. Am J Cardiol 1987; 59: 256-262.

36. Haley VB, Talbot TO, Felton HD. Surveillance of the short-term impact of fine particle air pollution on cardiovascular disease hospitalizations in New York State. Environ Health. 2009; 22: 8:42 